\title{
Growth and Characterization of Ge Nanocrystals
}

\author{
Soumyendu Guha \\ Mark Wall \\ Lloyd L. Chase
}

This paper was prepared for submittal to the European Materials Research Society (EMRS)

Strasbourg, France

June 15-19, 1998

May 1998

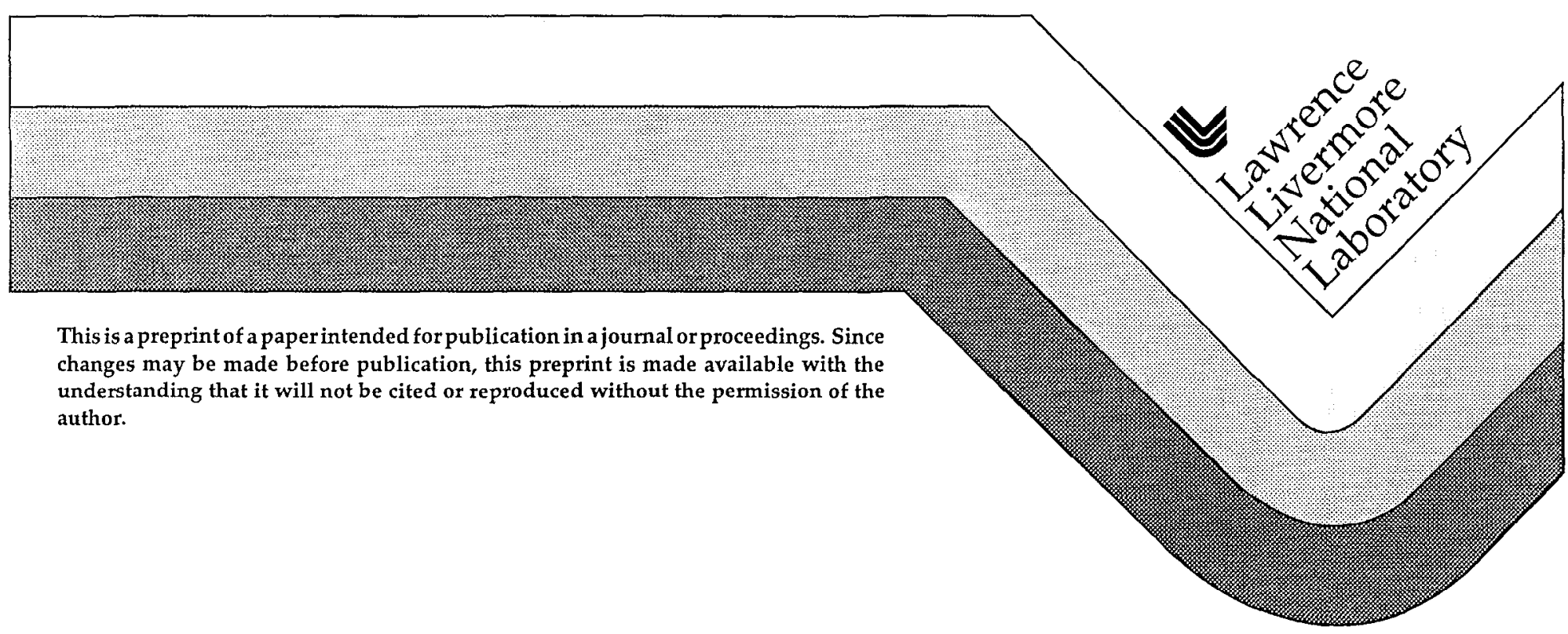




\section{DISCLAIMER}

This document was prepared as an account of work sponsored by an agency of the United States Government. Neither the United States Government nor the University of California nor any of their employees, makes any warranty, express or implied, or assumes any legal liability or responsibility for the accuracy, completeness, or usefulness of any information, apparatus, product, or process

disclosed, or represents that its use would not infringe privately owned rights. Reference herein to any specific commercial product, process, or service by trade name, trademark, manufacturer, or otherwise, does not necessarily constitute or imply its endorsement, recommendation, or favoring by the United States Government or the University of California. The views and opinions of authors expressed herein do not necessarily state or reflect those of the United States Government or the University of California, and shall not be used for advertising or product endorsement purposes. 
(To be presented at the European Materials Research Society

(EMRS) meeting at Strasbourg, France, June 15-19, 1998)

Session: J-VIII/P31

Growth and characterization of Ge nanocrystals

Soumyendu Guha

Naval Research Laboratory, Condensed Matter and Radiation Sciences

Division, Washington DC 20375

Guha@ccf.nrl.navy.mil

Mark Wall and Lloyd L. Chase, Chemistry and Materials Science Division, Lawrence Livermore Laboratory, Livermore, CA 94550.

We have synthesized Ge nanocrystals of sizes 4,8 , and $12 \mathrm{~nm}$ by ionimplanting $\mathrm{Ge}^{+}$ions into thermally grown $\mathrm{SiO}_{2}$ films and subsequent annealing of the films at $830^{\circ} \mathrm{C}$ for $30 \mathrm{~min}$ in nitrogen. These films were characerized by $x$-ray, transmission electron microscopy (TEM), and Raman spectroscopy. A distribution of particle size was identified by TEM in a $100 \mathrm{~nm}$ band below the surface. Particle sizes were estimated by these 3 techniques. 
Ion beam synthesis of semiconductor nanoparticles in thermally grown $\mathrm{SiO}_{2}$ films has recently drawn a considerable interest because of its ease and simplicity in growing particles of varying size. Both Si [1] and Ge[2-3] nanoparticles have been grown by ion-implanting either $\mathrm{Si}^{+}$or $\mathrm{Ge}^{+}$ions into thermally grown $\mathrm{SiO}_{2}$ films and subsequent annealing at temperatures $800^{\circ} \mathrm{C}$ or higher in a nitrogen environment. Due to their compatibility with microelectronics, both of these group IV compounds in the nanocrystalline phase offer a variety of applications due to their interesting optical [4] and electronic properties[5]. However, the growth of these nanoparticles with a uniform size distribution has been a challenge to the material scientists. A narrow uniform size distribution of these nanoparticles would enhance the physical properties of these materials which would eventually lead to their successful applications to optoelectronic devices.

In this report we describe the synthesis of Ge nanocrystals by ionimplanting $\mathrm{Ge}^{+}$ions into $500 \mathrm{~nm}$ thick $\mathrm{SiO}_{2}$ films and subsequent annealing. $\mathrm{SiO}_{2}$ films were grown by thermall oxdising a 4" Si wafer. These wafers were then cut into a size of $1 \mathrm{~cm}^{2}$ in which $\mathrm{Ge}^{+}$ions were implanted. Three different doses of $\mathrm{Ge}^{+}$ions at an energy of $190 \mathrm{keV}$ were used to grow different size nanocrystals. These doses were $1 \times 10^{17}(\mathrm{Ge} 1), 2 \times 10^{17}$ (Ge2), and $3 \times 1017$ (Ge3) ions $/ \mathrm{cm}^{2}$. Trim calculations [6] show a projected range of $250 \mathrm{~nm}$ below the surface with a maximum atomic density of $16 \%$ for Ge1 at a depth of $130 \mathrm{~nm}$. Ge nanocrystals were formed when the as-implanted samples were annealed at $830^{\circ} \mathrm{C}$ for $30 \mathrm{~min}$ in a nitrogen atmosphere. They were annealed at the 
same time. We then characterize these samples by $\mathrm{x}$-ray, high-resolution transmission electron microscopy (HRTEM), and Raman spectroscopy.

The XRD pattern from the annealed samples exhibited the (111), (220), and (311) reflections similar to crystalline $G e(c-G e)$ diffraction peaks but were broadened due the random distribution of Ge crystallites. Figure 1 shows the (111) Bragg-diffraction peak from the annealed films and a c- Ge wafer. The mean sizes were determined from the $2 \theta$ full-width at half-maximum (FWHM) of the (111) peak. The accuracy of this estimate is about $\pm 0.5 \mathrm{~nm}$.

Figure 2(a) shows a cross-section (CS) bright field TEM image of an annealed film. The low and high magnification images were obtained with JEOL electron microscopes (Model JEM 300CX and JEM 4000EX). In accordance with the TRIM calulation, Ge clusters were arranged in a band at a depth of $120 \mathrm{~nm}$ below the surface. The band boundary toward the surface shows larger crystallites (>10 nm), but smaller sizes were observed at a depth of $150 \mathrm{~nm}$. Figures 2(b) and (c) show HRTEM images of two annealed films . We show only a small $(2.1 \mathrm{~nm})$ and a large size $(7.8 \mathrm{~nm})$ nanocrystals from these annealed films. The lattice fringe spacings $(0.326 \mathrm{~nm})$ match that of the $\mathrm{c}-$ Ge. It is not clear why we observed a large precipitation of Ge clusters near the surface. We are currently conducting more investigation of the cluster formation by annealing these films in a temperature controlled high-vacuum furnace.

Figures 3(a) and (b) show a histogram of the particle-counts as a function of particle-size from these samples. These histograms were obtained from plan-view TEM images. The solid line is a fit to the histogram with a log-normal 
distribution function [7]. The estimated sizes from CS-TEM images were slightly higher and closer to the values obtained from $x$-ray (Fig. 1).

Figure 4(a) shows the phonon dispersion [8-9] of the transverse (TO) and longitudinal optical modes (LO) along the (100) direction as a function of the phonon wave vector $q$ in the first Brillouin zone. Note that $a$ is half the lattice constant for the cubic unit cell of a diamond lattice. The neutron data [8] are shown by three different symbols and the solid lines are cubic-polynomial fits to these data. It is important to point out that the phonon dispersion along the (110) and (111) directions are very similar [9] to the (100) direction except at the zone boundary. Figures 4(b) and (c) show the lineshapes of Raman spectra for the two optic modes as a function of particle size. The Raman line shape was obtained from the following formula [10],

$$
I=\int_{0}^{q} \frac{|c(0, q)|^{2} d^{3} q}{[\omega-\omega(q)]^{2}+\left[\frac{\Gamma}{2}\right]^{2}}
$$

where $\omega(q)$ is obtained from either the LO or the TO phonon dispersion curve shown in Fig. 4(a). The wave vector $q$ is expressed in units of $2 \pi / a$ where a (5.6 A) is the Ge lattice constant. The parameter $c(0, q)$ is estimated from the relationship:

$$
|c(0, q)|^{2}=\exp \left(-\frac{q^{2} L^{2}}{4 a^{2}}\right)
$$


where $L$ is the average size of a Ge nanocrystal. For a particle size of $300 \mathrm{~A}$ or higher, we fit the lineshape of the c-Ge phonon spectra by choosing $\omega$ and $\Gamma$ as $300.6 \mathrm{~cm}^{-1}$ and $7.5 \mathrm{~cm}^{-1}$, respectively. We then vary $L$ to obtain several Raman lineshapes shown by Figures $3(b)$ and (c).

The red shift of the Raman spectra as a function of particle size is caused by the decrease in phonon frequency $(\omega)$ as a function of $q$. Near the zone boundary the LO phonon frequency decreases considerably as compared to the TO phonon frequency. As a result one observes a considerable broadening of the LO spectra for smaller particles. The shift of the LO phonon frequency for a $20 \mathrm{~A}$ particle is about $7 \mathrm{~cm}^{-1}$ from the bulk phonon frequency, while that for the TO, mode is $12.6 \mathrm{~cm}^{-1}$.

Figure 4 (a) shows the Raman spectra from the three annealed samples along with a c-Ge sample. The shift of the phonon mode is about $2-3 \mathrm{~cm}^{-1}$ for all samples as compared to the phonon frequency $\left(300.6 \mathrm{~cm}^{-1}\right)$ of $\mathrm{c}-\mathrm{Ge}$. The Raman spectra were taken with a cooled CCD array of resolution of $1.7 \mathrm{~cm}^{-1} / \mathrm{pixel}$ at an excitation wavelength of $488 \mathrm{~nm}$. The uncertainty in the measurement of the peak position is roughly 1 pixel. The resolution of our spectrometer [10] is about $6 \mathrm{~cm}^{-1}$. With this kind of instrument resolution, it is difficult to estimate the particles sizes between 40 and $100 \mathrm{~A}$ by probing the shift and broadening of the phonon band of Ge nanocrystals. According to the theoretical model, the peak positions vary between 297 and $300 \mathrm{~cm}^{-1}$ when the sizes were varied between 40 and $100 \mathrm{~A}$. For Si nanocrystals [10], the phonon frequency was found to shift by about $10 \mathrm{~cm}^{-1}$ from the bulk phonon frequency 
$\left(521 \mathrm{~cm}^{-1}\right)$ for a particle of size $40 \mathrm{~A}$ and that's why Raman spectroscopy was useful in estimating the particle size of Si nanocrystals.

Figures 4(b) and (c) show a fit to the lineshape of the Raman spectra of samples $\mathrm{Ge1}, \mathrm{Ge2}$, and $\mathrm{Ge} 3$. A shoulder at $280 \mathrm{~cm}^{-1}$ was observed in all samples that we attribute to amorphous Ge phonon band [9]. The lineshape (solid line) was obtained from the theoretical model using Eqn. (1). As we anticipated, we measure approximately the same size for the three annealed samples since we found the shift and broadening of the spectra identical in all three samples. The only explanation that we can offer for measuring the same sizes in all samples is the instrument resolution. The shift and broadening of the phonon band are not large enough to be discerned by our instrument resolution. Note that in reference 11, significant shift and broadening of the phonon spectra were only observed for particles of size less than $15 \mathrm{~A}$.

In summary, we have synthesized three sizes of Ge nanocrystals grown by ion-implanting $\mathrm{Ge}^{+}$ions into $\mathrm{SiO}_{2}$ films and subsequent annealing. We estimated the mean particle size of the annealed samples to be 4,8 , and $12( \pm 1$ $\mathrm{nm}$ ) by $\mathrm{x}$-ray diffraction and HRTEM.

We acknowledge Mr. Randy Walker of NRL for ion-implantation, Mr. Dominic Del Giudice of LLNL for x-ray measurements and Dr. I. L. Singer of NRL for TRIM calculations. 


\section{References}

1. S. Guha, M. D. Pace, D. N. Dunn, and I. L. Singer, Appl. Phys. Lett. 70, (1997) 1207. Please also see other references therein.

2. K. S. Min, K. V. Scheglov, C. M. Yang, H. A. Awater, M. L. Brongersma, and A. Polman, Appl. Phys. Lett. 68, (1996) 2511.

3. J. von Borany, R. Grotzschel, K. H. Heinig, A. Markwitz, W. Matz, B. Schmidt, and W. Skorupa, Appl. Phys. Lett. 71, 3215 (1997). Also see other references therein.

4. R. T. Collins, P. M. Fauchet, M. A. Tischler, Physics Today, 50 (1997) 24.

5. Y. Maeda, Phys. Rev. B 51, (1995) 1658. Also see other references therein. 6. J. F. Ziegler, J. P. Biersack, and U. Littmark, The stopping and Range of ions in Solids (Pergamon, New York, 1985).

7. H. Yorikawa and S. Muramatsu, Appl. Phys. Lett 71, (1997) 644.

8. G. Nilsson and G. Nelin, Phys. Rev B 3, (1971) 364.

9. Y. Sasaki and C. Horie, Phys. Rev B 47, (1993) 3811.

10. S. Guha, P. Steiner, and Walter Lang, J. Appl. Phys., 79 (1996) 8664.

11. C. E. Bottani, C. Mantini, P. Milani, A. Stella, P. Tognini, P. Cheyssac, and R. Kofman, Appl. Phys. Lett 69, (1996) 2409. 


\section{Figure Caption}

Figure 1. x-ray diffraction spectra of three annealed and a c-Ge samples. The peak at $26.5^{\circ}$ has been identified as the $\mathrm{GeO}_{2}$ phase. The broad peak at $27.35^{\circ}$ has been identified as the cubic phase of Ge.

Figure 2. (a) Cross-section TEM image of the $\mathrm{SiO}_{2}$ layer of an annealed sample. The Ge clusters can be identified by the dark spots. HRTEM images of samples (b) Ge1 and (c) Ge2. The (111) lattice planes of Ge clusters can be easily seen.

Figure 3. Histograms (shaded areas) of size distributions from the plan-view images of two annealed samples. The solid line is a fit with a log-normal function. The mean diameters of particles are expressed as $D_{1}$ and $D_{2}$.

Figure 4. (a) Dispersion curves for the three optic modes of c-Ge. Selective neutron datapoints are shown by the symbols, while the solid line is a cubic polynomial fit to the data. Raman lineshapes for the (b) LO and (c) TO modes were calculated for different sizes.

Figure 5. (a) Raman spectra of three annealed and a c-Ge sample in a backscattering geometry. A vertically polarized incident laser beam with laser power of $10 \mathrm{~mW}$ was focused into a $300 \mu$ spot. The scattered beam was unpolarized. (b) and (c) Theoretical fits to the experimental Raman data. Note that a similar $50 \mathrm{~A}$ fit is also obtained for the $\mathrm{TO}_{2}$ mode and is not shown. 


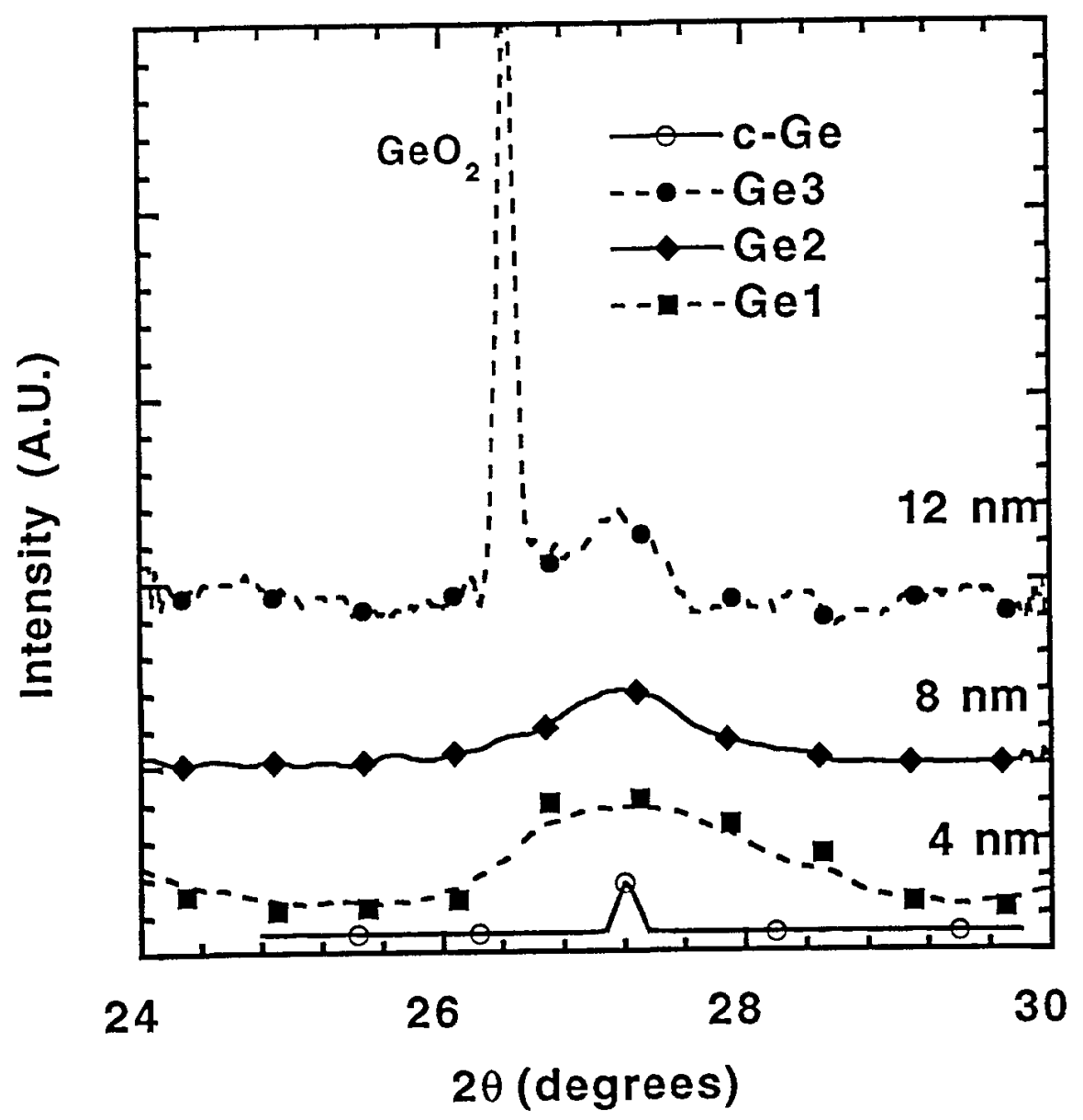




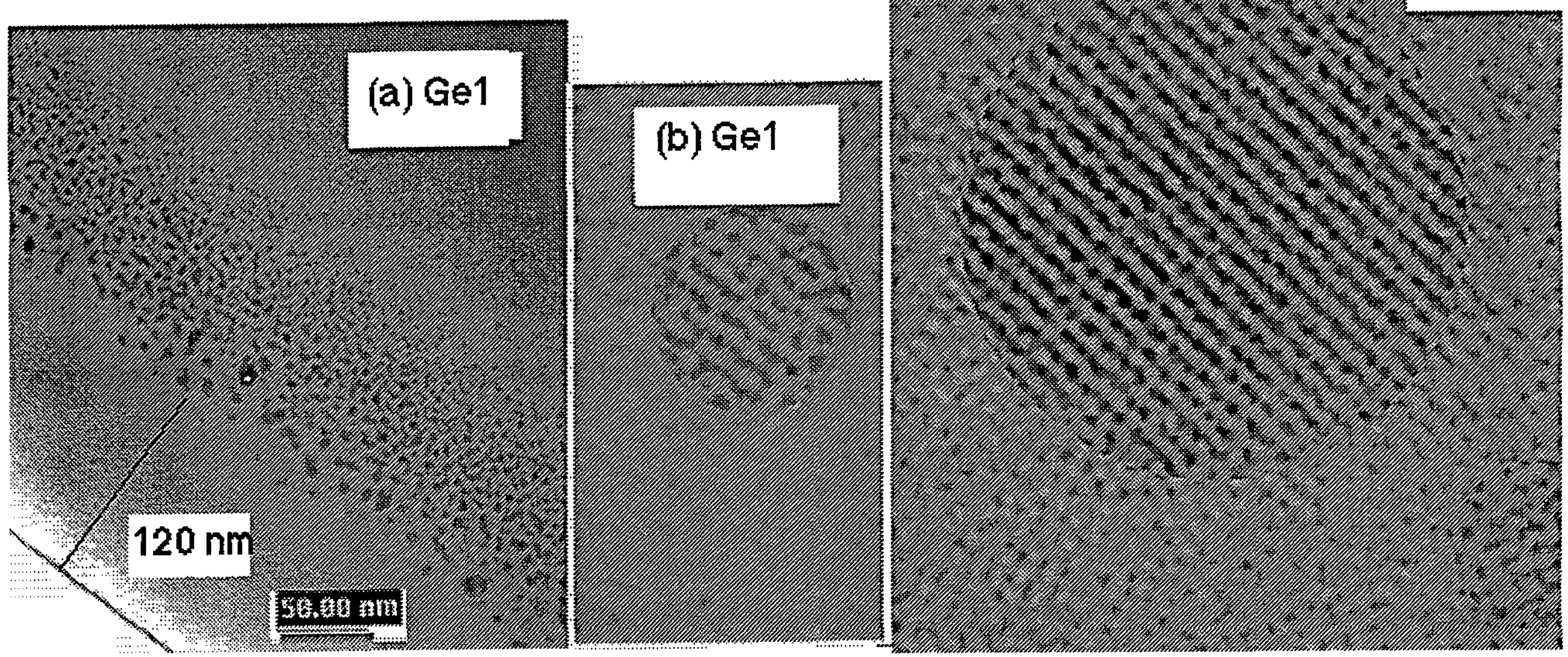



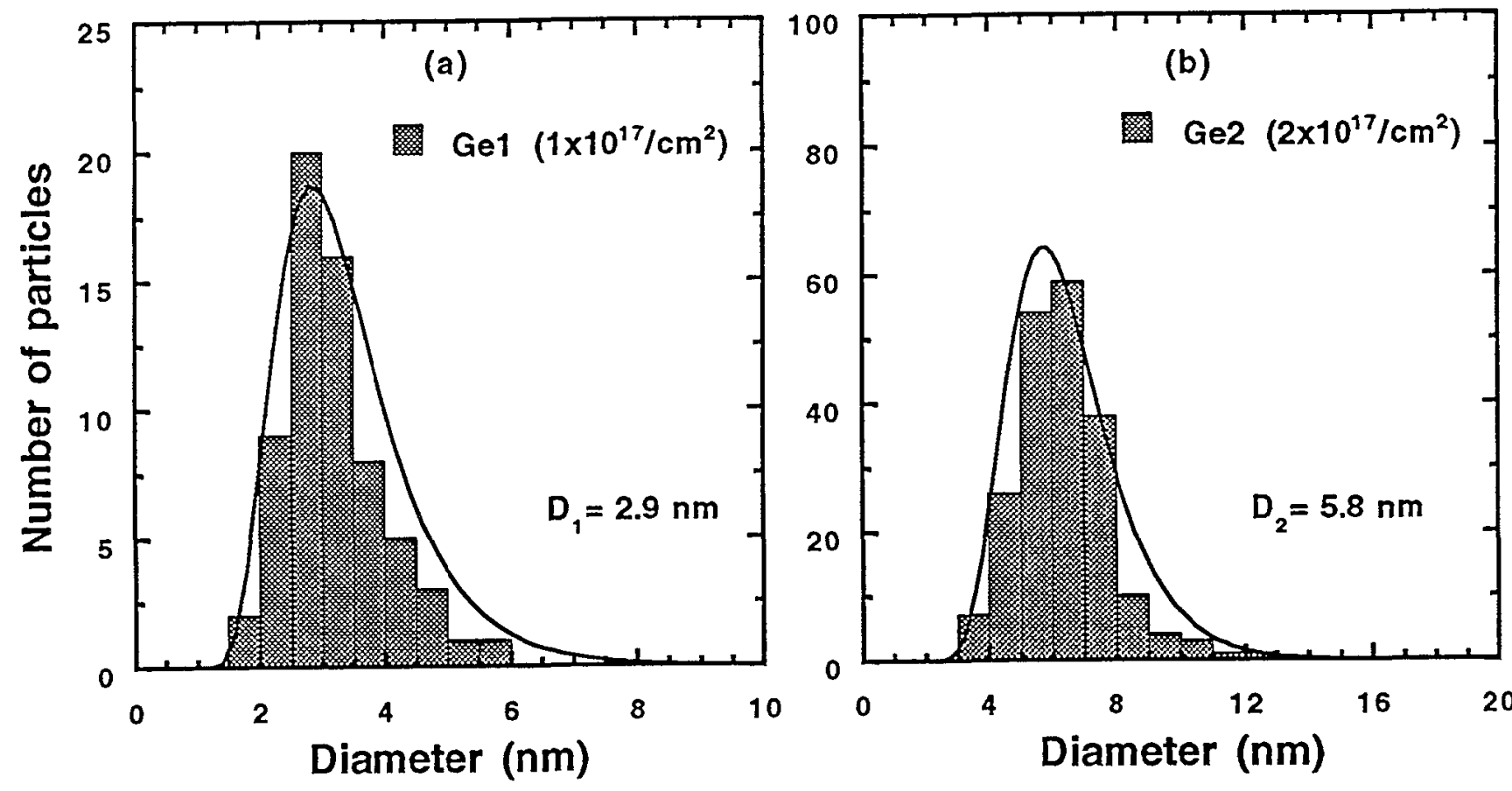

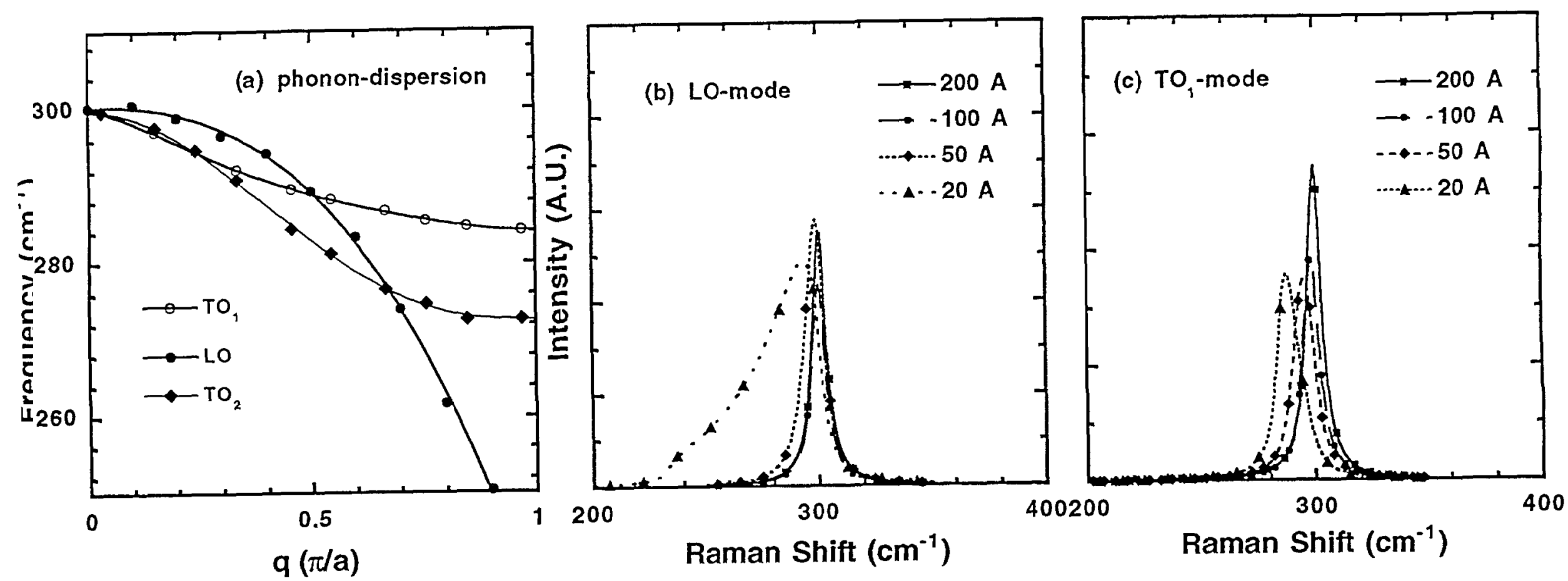

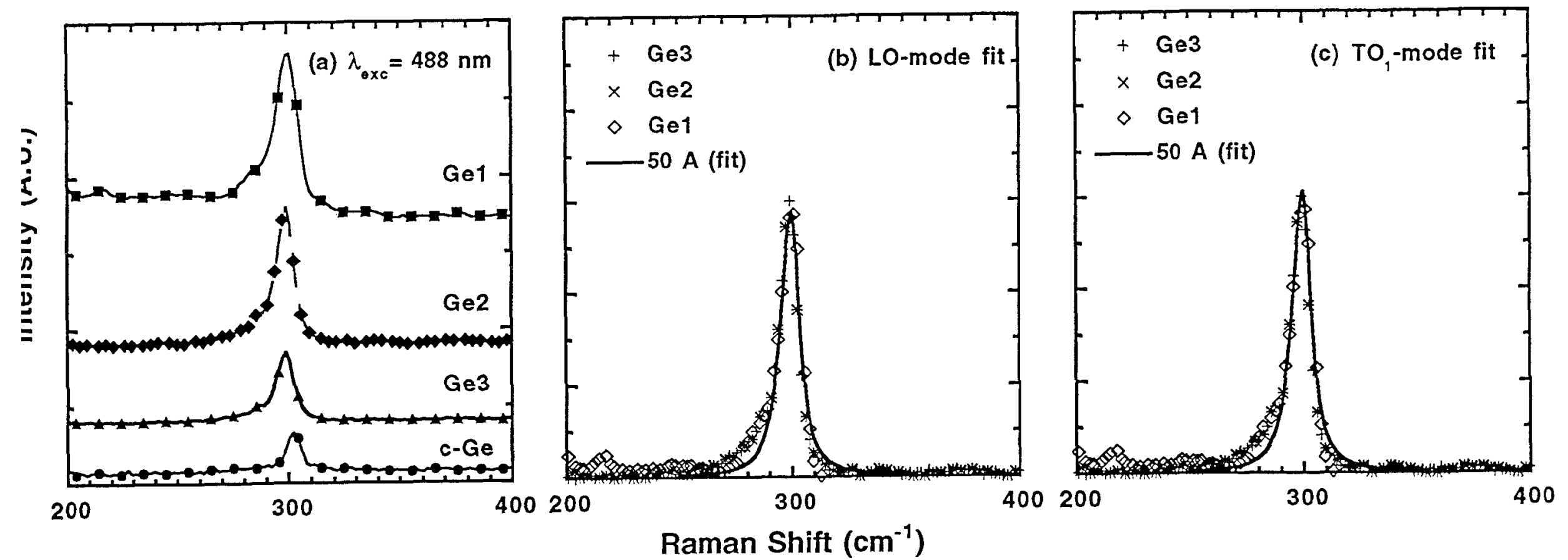


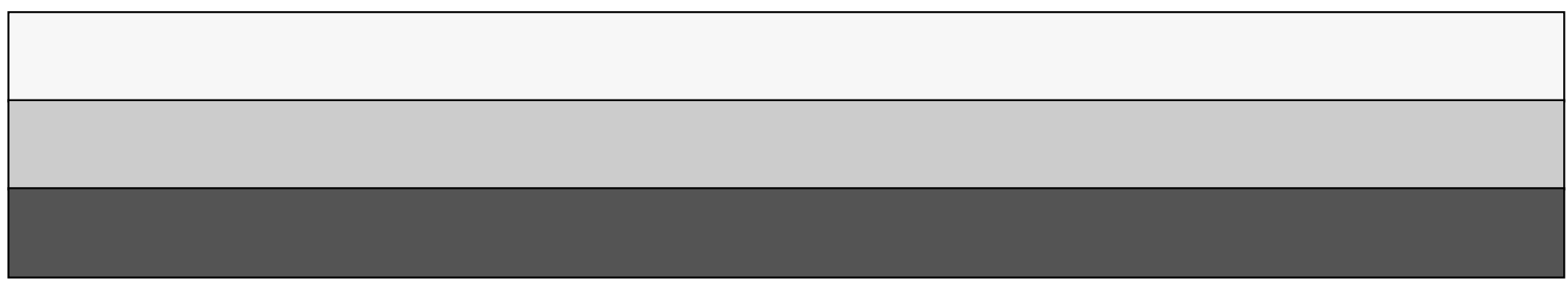

